\section{FATHERS MATTER TOO: INVOLVING BOTH FATHERS AND MOTHERS IN CHILD HEALTH RESEARCH}

\author{
J. Price ${ }^{1}$, J. Jordan², L. Prior ${ }^{3}$, J. Parkes ${ }^{2}$
}

${ }^{1}$ Queen's University Belfast, ${ }^{2}$ School of Nursing and Midwifery, ${ }^{3}$ School of Sociology, Social Work and Social Policy, Queen's University Belfast, Belfast, UK

Background: Current research within the field of children's palliative care primarily focuses on the perspectives of mothers, to the relative detriment of fathers. The reason for the absence of the male perspective has often been attributed to the difficulty of recruiting fathers and their unwillingness to talk, particularly about sensitive subjects. Given the expansion of children's palliative care over recent years, the needs to understand how fathers experience caring for their dying child and their perspective on how this care can be facilitated professionally is ever more acute. Such understanding will support health and social care professionals to ensure that the care they provide for children is family centred and addresses the needs of both parents.

Conclusions: This poster examines the pertinent issue of involving bereaved fathers in qualitative research interviews and considers the practical issues of such involvement. Little guidance exists regarding whether it is best to interview parents together or separately. The PATCH study used semistructured interviews to examine bereaved parents' experiences of caring for their child at the end-oflife. Some parent couples were interviewed jointly, others separately. Advantages and disadvantages of both approaches will be outlined and excerpts from parent interviews will be used to substantiate points made.

\section{7}

\section{THE OYGEN TISSUE SATURATION (SPO2) AUDIT}

\section{Jones}

\section{Paediatric Intensive Care, Birmingham Children's} Foundation NHS Trust, Birmingham, UK

Background and aims: Pulse oximetry $(\mathrm{SpO} 2)$ is a fundamental aspect of PIC (Paediatric Intensive Care) (Hanning and Alexander-Williams, 1995). Due to widespread use of pulse oximetry, competence and thus understanding of the basic principles related to this monitoring is vital. Clinical audit was performed to ascertain nursing knowledge related to the use of pulse oximetry.

Method: A questionnaire was given out to nurses $(n=40)$ at a PIC unit. The questionnaire contained four questions regarding the use of pulse oximetry. The results were counted using nominal data and content analysis was used to ascertain themes.

Results: Less than half of those who participated felt that pulse oximetry was indicated in children who had respiratory distress (45\%), respiratory failure $(10 \%)$ or those who were requiring supplemental oxygen therapy (40\%).

$80 \%$ of participants could explain how pulse oximetry works. Common themes from content analysis were infrared light versus light meeting a sensor through the periphery and detecting the amount of oxygen bound to haemoglobin. $20 \%$ could not explain how pulse oximetry worked.

Disadvantages highlighted were burns related to use $(35 \%)$ and inaccuracy on movement (55\%).

Rotation of probe sites (82\%) was listed as the most common nursing consideration when using pulse oximetry. Pressure sore and burn prevention, was also found to be paramount.

Conclusion: Results suggest that further education regarding the use of pulse oximetry is needed. Education regarding the indications for the use of this monitoring is required. Further, more indepth investigation is required.

\section{8}

\section{MAKING A DIFFERENCE: INTRODUCING PAEDIATRIC CRITICAL CARE OUTREACH SUPPORTS EFFECTIVENESS OF AN EARLY WARNING SCORING SYSTEM IMPROVING PATIENT SAFETY}

\author{
N. Taylor, S. McHale \\ PICU, Nottingham Children's Hospital, \\ Nottingham, UK
}

Nottingham Children's Hospital has recently developed and introduced an integrated Paediatric Early Warning Scoring tool and Observation Chart. To support this development and to enable the structured escalation plan to be instigated, a PCCOT team was also introduced and trialled for one month. 